\title{
Chloriodide of potassium
}

\section{Filhol}

To cite this article: M. Filhol (1839) Chloriodide of potassium, Philosophical Magazine Series 3, 15:99, 555-557, DOI: 10.1080/14786443908649959

To link to this article: http://dx.doi.org/10.1080/14786443908649959

册 Published online: 01 Jun 2009.

Submit your article to this journal

Џ Article views: 3

Q View related articles $₫$ 
is deposited from solutions containing free sulphuretted hydrogen; the more they contain of this the whiter is the sulphur. It is never whiter than when it is deposited from water saturated with sulphuretter hydrogen, in which the hydrogen of the sulphuretted hydrogen has been gradually oxidized by the contact of the air. If on the contrary finely divided sulphur be precipitated from solutions which do not contain free sulphuretted hydrogen, it has a yellow colour, even when the quantity is small. It is sufficient to decompose a small quantity of a solution of an alkaline hyposulphite by an acid, to be convinced of the truth of this assertion.

White or gray precipitated sulphur, (milk of sulphur) contains a very small quantity of sulphuretted hydrogen, in the state of persulphuret of hydrogen. If it be fused and the small quantity of gas disengaged from the surface of the fused sulphur be conducted by the aid of a current of atmospheric air into a solution of lead, a notable quantity of sulphuret of lead is obtained. M. Rose treated in this way a large quantity of the different nodifications of this white sulphur prepared in different modes, and he always obtained the same result.

In melting flowers of sulphur, or roll sulphur, it is true that there is sometimes obtained a little sulphuretted hydrogen; but the quantity of it is so small that it cannot be compared with that disengaged from the milk of sulphur. Water cannot remove from this lastmentioned substance the small quantity of sulphuretted hydrogen which it contains; for all the modifications examined were washed with water till it produced no effect in a solution of lead.-Journal de Pharmacie, Aout, 1839.

\section{URIC ACID.}

M. Fritzsche has analysed hydrated crystallized uric acid. When the process given by $M$. Boeteger of preparing uric acid is adopted, and which consists in dissolving pigeons' dung in a solution of borax, and precipitating the uric acid with hydrochloric acid, the acid is procured in much larger crystals when the solution contains a great quantity of organic matter than when it does not contain any. The separation of the uric acid takes place readily, and but a small quantity remains in the liquor. Even this separates on standing, in the form of yellowish brown dendritic crystals, of a line in length, and are hydrated uric acid. This hydrate, when dried at $212^{\circ}$ Fahr., loses about 21.52 per cent. of water. It is only these large crystals which are hydrated; whenever it is precipitated from hot dilute solutions the acid is always of this kind; the smaller the crystals are, the more readily they part with a portion of their water at the usual temperature, and it is on this account that they have been so long unknown.-L'Institut, No. 304.

\section{CHLORIODIDE OF POTASSIUM. BY M. FILHOL.}

When a current of chlorine gas is passed through perfectly neutral iodide of potassium dissolved in twice its weight of water, the first bubbles of the gas communicate a brown colour to the liquor; the 
intensity of this colour increases; the liquor soon becomes opake, and iodine is precipitated in a pulverulent state. If the operation be stopped when this deposit ceases to be formed, all the iodine is separated, no sensible quantity of chloride of iodine is formed, and the liquor contains chloride of potassium ; if the operation be continued, the iodine redissolves, and the liquor assumes a fine golden colour. Sometimes a little chloriodate of potash precipitates; this happens especially if the iodide is not perfectly neutral, and it is casy to account for it. When all the iodine is dissolved, it is proper to continue the disengagement of the chlorine: fine golden coloured needles soon appear in the liquor, and the quantity increases as the saturation with the chlorine proceeds. When a saturated solution of the iodide is employed, the liquor submitted to the action of the chlorine, is eventually converted into a yellow needle-formed mass of a beautiful appearance; in order that the reaction may be perfect, it is well to heat the liquor slightly, that the tube which conveys the chlorine may not be obstructed.

When the chlorine ceases to be sensibly absorbed, the operation is to be stopped, and then, in order to obtain finer crystals, the bottle may be immersed in water heated from $105^{\circ}$ to $125^{\circ} \mathrm{Fahr}$; by this the crystals are redissolved, and recrystallization takes place slowly. The crystals are similar to those obtained by the action of hydrochloric acid on iodate of potash ; but the process now described is very convenient for obtaining considerable quantities of this salt.

It appears to be composed of

One equivalent of bichloride of iodine...... 234

One $", \quad$ chloride of potassium ..... $76-310$

and when it is heated the perchloride of iodine is expelled and the chloride of potassium remains.

The iodides of ammonium and of magnesium furnished results corresponding to the above; but the iodides of sodium and barium did not yield chlorosalts. Biniodide of mercury was treated in the same manner, and a fine golden-coloured solution was obtained which did not yield crystals.

It will be observed that chlorine acts upon the iodides as it does upon the sulphurets; but the chloride of sulphur combines readily with acid chlorides, whilst the chloride of iodine combines better with the alkaline chlorides.

It ought to be observed, that the salt obtained by the process above described, if it be required very pure, ought to be very quickly separated from the watery liquid, in which it is formed; for if the chloride of iodine is decomposed in iodic and hydrochloric acids, which may happen, as is well known, with great readiness, it may occur that the less solubility of the iodate of potash may determine the decomposition of the alkaline chloride, and the precipitation of a little iodate, for there does exist sufficient hydrochloric acid to prevent the formation of the iodate which occurs rapidly; the salt therefore intended for analysis ought to be separated as soon as it is formed. 
The addition of hydrochloric acid is even indispensable to prevent also the precipitation of the salt discovered by Serullas, the crystals of which are readily distinguished by the unassisted eye.-Journal de Pharmacie, Aout, 1839.

FALL OF A METEORITE IN MISSOURI, FEBRUARY 13, 1839.

On the afternoon of the 13th of February, 1839, a meteor exploded near the settlement of Little Piney, Missouri, (lat. $37^{\circ} 55^{\prime}$ N. ; lon. $92^{\circ} 5^{\prime} \mathrm{W}$.) and cast down to the earth one stony mass or more in that vicinity. Mr. Forrest Shepherd, of this city, who was at the time exploring this region in the line of his profession, viz. that of a mineralogical and geological surveyor, hearing of the explosion of the meteor, exerted himself to collect all the circumstances of the occurrence. He subsequently succeeded in obtaining several fragments of one of the stones thrown down by the meteor. Mr. Shepherd has favoured me with an opportunity to examine these fragments, and has also communicated to me the details below related.

The meteor exploded between 3 and $40^{\prime}$ clock P.M., of the 13th of February, 1839, and although the sky was clear, and the sun of course shining at the time, the meteor was plainly seen by persons in Potosi, Caledonia, and other towns near which it passed. At Caledonia, which is about nine miles south-westerly from Potosi, the meteor passed a little north, and at the latter place, a little to the south of the zenith. Its course was almost precisely to the west. The most eastern spot at which it was seen is about fifteen miles west of St. Genevieve, (or about lat. $375_{6}^{\circ} \mathrm{N}$.; lon. $90^{\circ} \mathrm{W}$.) -the most western is Little Piney, near which it exploded. To the observers at the latter place, the meteor appeared of the size of a large star. They represent its motion as very slow; but do not state how many seconds it was in sight. We have no data for determining the meteor's size, or velocity, or the inclination of its path to the horizon. The direction of the meteor's motion with regard to that of the earth, was probably such that the velocity of the former would be apparently diminished; and as at Little Piney the meteor must have traversed only a small arc, its motion, to an observer there, would appear quite slow. At the time of the occurrence, Mr. Shepherd was on the western bank of the Mississippi, near St. Mary's landing, and heard a distant report, which he was afterwards inclined to refer to the explosion of this meteor. At Little Piney, Mr. Harrison and others saw the meteor burst in pieces, and in a minute or a minute and a half afterwards, they heard three explosions in quick succession. Some of the inhabitants went in quest of the stones which they supposed had fallen, and finally found a tree which appeared to have been recently injured by the collision of some solid body. Near this tree they discovered (although the ground was covered with three or four inches of snow, one of the meteoric stones, about as large as a man's head, partly imbedded in the earth; and from the circumstances of its position and appearance, there could be no reasonable doubt that this was the body 